\title{
¿SE J USTIFICA LA OOFORECTOMÍA, COMO MEDIDA PROFILÁCTICA EN LA DISMINUCIÓN DEL RIESGO DE CÁNCER DE OVARIO Y DE MAMA, EN LAS PACIENTES PORTADORAS DE MUTACIONES EN LOS GENES BRCA1 O BRCA2?
}

\author{
Marcelo González $V^{1}$, Demetrio Larraín de la C. ${ }^{a}$, Maximiliano Figueroa M. ${ }^{a}$ \\ ${ }^{1}$ Servicio de Cirugía, Instituto Nacional del Cáncer, Universidad de los Andes.
}

alnternos de Medicina, Universidad de los Andes

\begin{abstract}
RESUMEN
Los avances en genética hacen necesario que los médicos clínicos conozcan las implicancias y repercusiones que tiene en la vida de la mujer el ser portadora de las diferentes mutaciones de genes supresores de tumor (BRCA1 o BRCA2). Esta revisión busca la evidencia científica que ayude al consejo de las pacientes, para sugerir distintas alternativas de tratamiento, como son el seguimiento estricto y la ooforectomía profiláctica. La evidencia publicada hasta el momento, permite una adecuada guía a clínicos y pacientes, tanto en aquellas portadoras de mutaciones genéticas, como en las que solicitan consejo por dudas y temores relacionados con la aparición de cáncer de ovario. Basados en la evidencia publicada, se concluye que la ooforectomía profiláctica disminuye el riesgo de aparición de cáncer de ovario y de mama en mujeres portadoras de mutaciones en los genes BRCA1 y BRCA2.
\end{abstract}

PALABRAS CLAVES: Cáncer de ovario, ooforectomía profiláctica, genes BRCA

\section{SUMMARY}

With the advance of genetics, it is necessary that the physicians know the importance for the women to carry a BCRA1 and BRCA2 mutations. An extensive review about the evidence of prophylactic oophorectomy in these patients is performed. Currently, the experience published is enough for the development of clinical guidelines for patients who carry a BRCA1 and BRCA2 or if they are looking for an advice in relation to the risk of develops ovarian cancer. Based on evidence, prophylactic oophorectomy does protect against the development of ovarian and breast cancer in women who carry BCRA1 and BRCA2 mutations.

KEY WORDS: Ovarian cancer, prophylactic oophorectomy, BRCA mutations

\section{INTRODUCCIÓN}

Las mujeres portadoras de mutaciones en los genes BRCA1 o BRCA2 tienen un aumento signi- ficativo en el riesgo de cáncer de mama y de ovario en comparación con la población general. Estas mujeres tienen un riesgo acumulado de cáncer invasor de mama a lo largo de su vida que 
oscila entre 55 y $85 \%$ y de cáncer epitelial invasor de ovario entre 15 y $65 \%$ (1-4). Se estima así que el riesgo acumulado de desarrollar cualquiera de los cánceres a lo largo de la vida es, en consecuencia, cercano al $100 \%(5,6)$.

BRCA1 y BRCA2 son genes supresores de tumor que en condiciones fisiológicas inhiben el crecimiento de células tumorales. Ambos genes son potentes activadores de la transcripción y codifican para proteínas con múltiples funciones, pero su rol en la reparación del DNA dañado parece ser lo más importante en la carcinogénesis $(3,4,7)$.

La identificación de los genes BRCA y la disponibilidad de pesquisa genética para las mutaciones de éstos, han puesto énfasis en la necesidad de mejorar la prevención y detección temprana del cáncer de mama y ovario (8-11).

Es esencial para cualquier recomendación clínica el conocimiento de la probabilidad de desarrollo de cáncer en el portador de una mutación determinada. En el caso de los genes BRCA, éstos tienen penetración incompleta y la probabilidad de desarrollar cáncer es variable $(2,12)$. Las portadoras de una mutación BRCA1 tienen entre un 50 a $85 \%$ de riesgo de desarrollar cáncer de mama y un 20 a $40 \%$ de desarrollar cáncer de ovario en el transcurso de su vida; mientras que las con mutación del BRCA2 parecen tener un riesgo similar para cáncer de mama y un 10 a $20 \%$ de riesgo para el cáncer de ovario, inferior al otorgado por el BRCA1 mutado $(1-5,13)$.

El cáncer familiar tiende a aparecer en mujeres más jóvenes que el cáncer esporádico, con un mayor riesgo, pues éste se mantiene de por vida en portadoras de estas mutaciones, desarrollando en algunos casos cáncer bilateral de mama o tumores de ovario y mama en forma simultánea $(4,14)$. Aunque el riesgo de cáncer ovárico en portadoras de mutaciones de BRCA es considerablemente menor que el riesgo para cáncer de mama, la ausencia de métodos confiables de detección temprana y la letalidad del cáncer ovárico avanzado han llevado a muchos oncólogos a recomendar la ooforectomía profiláctica bilateral luego de que la mujer haya completado su fertilidad. Sin embargo, la evidencia a favor de una disminución significativa del riesgo de cáncer otorgada por el procedimiento es bastante limitada, y sólo se basa en escasos estudios retrospectivos y en la opinión de expertos (15, 16-19).

El objetivo de este estudio es revisar si existe suficiente evidencia, que permita aceptar que la ooforectomía bilateral profiláctica es una medida justificada y efectiva en la reducción del riesgo de cáncer de mama y ovario en pacientes portadoras de mutaciones en los genes BRCA1 o BRCA2.

\section{MATERIAL Y MÉTODO}

Se inició una búsqueda en tres diferentes bases de datos disponibles en Internet: MEDLINE, MDConsult y Cochrane Data Base. El criterio utilizado para seleccionar la información, fue el de limitar nuestra búsqueda a todas aquellas publicaciones que relacionaran en sus resúmenes, títulos o textos, las palabras claves BRCA mutations, ovarian cancer, breast cancer y prophylactic oophorectomy, entre los años 1997 y 2004. Se revisó también la bibliografía de estos artículos en busca de referencias relevantes, que incluyeran artículos controlados aleatorios o metaanálisis que comparen ooforectomía profiláctica con otra estrategia para la prevención de cáncer de ovario en mujeres con mutaciones en los genes BRCA 1 ó 2 confirmadas o ensayos fase II, estudios de cohorte o series de casos que examinen los resultados de la ooforectomía profiláctica en mujeres con mutaciones en los genes BRCA 1 ó 2 confirmadas. Los resultados de esta búsqueda fueron los siguientes: Cochrane Data Base: 0 referencias; MEDLINE: 23 referencias; MDConsult: 32 referencias.

De los 55 artículos, 6 cumplían con nuestros criterios. Las 6 publicaciones seleccionadas fueron: Kauff y cols (20), Rebbeck y cols (21) y 4 modelos teóricos de análisis decisión (22-25).

\section{RESULTADOS}

Kauff y cols (20) reportan los resultados de un estudio prospectivo del efecto de la ooforectomía en la reducción del riesgo de cáncer de mama y ovario en 170 portadoras de mutaciones BRCA, con un seguimiento promedio de 2 años. El cáncer ovárico o un carcinoma papilar seroso del peritoneo se desarrolló en 5 de 72 mujeres quienes escogieron vigilancia intensiva $(6,9 \%)$. De las 98 que eligieron ooforectomía bilateral profiláctica, tres tenían tumores en etapas tempranas que fueron diagnosticados al momento de la cirugía $(3,1 \%)$; una paciente desarrolló cáncer peritoneal primario durante el seguimiento (1\%). El tipo de tumor que se desarrolla posteriormente sería considerado como derivado de los remanentes müllerianos en las líneas mesoteliales del peritoneo, cuya presencia está asociada con riesgo persistente de cáncer luego de la ooforectomía. Para evaluar el riesgo de cáncer de mama, las pacientes mastectomizadas fueron excluidas. Entre las 
mujeres no mastectomizadas, se observó un desarrollo de cáncer de mama en 8 de 62 mujeres sometidas a vigilancia estricta (12,9\%) y en 3 de 69 mujeres en el grupo de ooforectomizadas $(4,3 \%)$. Los análisis revelaron que el cociente de riesgo para el desarrollo de cáncer de mama o cáncer ginecológico relacionado al BRCA después de la ooforectomía profiláctica se reducía a 0,25 (95\% intervalo de confianza 0,08 a 0,74 ). No hubo diferencias significativas según el tipo de mutación (BRCA1 vs BRCA2) con relación al tiempo para desarrollar cáncer de mama u ovario $(p=0,31)$.

En un artículo complementario, Rebbeck y cols (21) reportaron resultados de un análisis de casos y controles retrospectivo multicéntrico de $551 \mathrm{mu}$ jeres portadoras de mutaciones BRCA1 o BRCA2. Entre las 292 mujeres que escogieron vigilancia estricta (examen ginecológico, ecografía transvaginal y medición de CA125 cada 6 meses) (controles), 58 (19,9\%) desarrollaron cáncer ovárico durante un promedio de seguimiento de 8,8 años. En contraste, entre las 259 mujeres quienes eligieron la ooforectomía profiláctica bilateral (casos), 6 de ellas presentaron tumores ováricos en etapa I al momento de la cirugía $(2,3 \%)$, y el cáncer peritoneal primario subsecuente se desarrolló en otros 2 casos $(0,8 \%)$. Con la exclusión de las 6 mujeres ya mencionadas, en las cuales la ooforectomía fue terapéutica, la cirugía profiláctica redujo significativamente el riesgo para cáncer ovárico derivado del epitelio celómico (cociente de riesgo 0,$04 ; 95 \%$ intervalo confianza 0,01 a 0,16 ). Durante 11 años de seguimiento, el cáncer de mamá fue diagnosticado en 60 de 142 mujeres sin historia de cáncer de mama bajo vigilancia estricta (42,3\%), comparados con 21 de 99 casos similares entre quienes eligieron ooforectomía profiláctica $(21,2 \%)$. De esta forma, el riesgo de desarrollar cáncer de mama fue significativamente menor a favor de la cirugía profiláctica (cociente de riesgo 0,$47 ; 95 \%$ intervalo de confianza 0,29 a 0,77 )

Estos 2 estudios apoyan la práctica habitual de recomendar la ooforectomía bilateral profiláctica a mujeres portadoras de mutaciones en los genes BRCA. Sus resultados son concordantes con estudios previos, más pequeños y con modelos de análisis de decisión (22-25) en los cuales los investigadores, mediante la construcción de modelos matemáticos, calcularon que mujeres con edad promedio de 30 años, portadoras de mutaciones en los genes BRCA, la ganancia en expectativa de vida para las ooforectomizadas variaba entre 0,3 y 9,5 años, dependiendo de su riesgo acumulativo de cáncer. A su vez se observó que esta ganancia disminuye con el aumento de la edad al tiempo de la cirugía y que es mínima para mujeres mayores de 60 años. Así, en mujeres de 30 años la ooforectomía profiláctica podría diferirse en 10 años con poca pérdida de la expectativa de vida.

\section{DISCUSIÓN}

Los resultados descritos, concluyen que la ooforectomía profiláctica es útil en la prevención del cáncer ovárico y de mama en portadoras de mutaciones en los genes BRCA, disminuyendo el riesgo para el desarrollo de estos tumores en un $95 \%$ y $50 \%$ respectivamente, tomando en cuenta que las mutaciones en los genes BRCA están presentes entre 0,1 a $0,2 \%$ de la población y que tienen un riesgo acumulativo de desarrollar cáncer de ovario y de mama en 15 a $65 \%$ y 60 a $80 \%$, respectivamente. Ambos estudios coinciden además, en el riesgo persistente del desarrollo de tumores peritoneales primarios, debido a que ovarios, trompas de Fallopio y peritoneo comparten esa línea celular, con un riesgo remanente de 4 a $5 \%$ para el desarrollo de esos tumores. Con esto queda de manifiesto que si bien la ooforectomía disminuye el riesgo de cáncer de tumores epiteliales, no lo elimina totalmente $(15,19)$. Este riesgo aumentado no estaría confinado sólo al ovario, sino que se extendería también a estructuras embriológicamente relacionadas con él como el tejido peritoneal y las trompas de Falopio (26). En pacientes con mutaciones en los genes BRCA las tubas presentan frecuentemente cambios displásicos, por lo que para disminuir aún más el riesgo de cáncer, la salpingooforectomía bilateral, probablemente con histerectomía, sería el procedimiento de elección y no sólo la ooforectomía (27-29).

Es importante destacar que la incidencia de cáncer ovárico en los controles del estudio de Rebbeck fue algo mayor que lo predicho en modelos epidemiológicos, y que los casos en mujeres con mutaciones en BRCA1 no fueron diferenciados de aquellas con mutaciones en BRCA2, los cuales tienen menor riesgo de cáncer ovárico. Considerando que el diagnóstico de cáncer ovárico se realiza con un promedio de edad de 50,8 años (rango de 30 a 73 años), en coincidencia con otras series $(12,30)$ se recomienda, según éste y otros autores, postergar la ooforectomía hasta completada la fertilidad, o realizarla entre los 35 y 40 años. Cumplidos estos plazos no debería diferirse sino efectuarse la cirugía lo antes posible (31). Esta recomendación se basa en el hallazgo 
de tumores ováricos en etapas tempranas en una fracción importante de las ooforectomizadas. Sin embargo, esto puede ser problemático en el caso del cáncer de mama hereditario, pues éste se desarrolla más temprano. De hecho, al observar en detalle el estudio de Kauff y cols se observa que el cáncer de mama ligado a mutaciones en genes BRCA ya se ha desarrollado en el $70 \%$ de las mujeres cuando la ooforectomía es postergada hasta los 47,5 años. Por eso, la recomendación de la cirugía profiláctica relacionada al promedio de edad de aparición de los tumores puede ser discutible, ya que se debe considerar que el cáncer hereditario puede variar en su tiempo de aparición según el tipo de mutación, lo que a su vez puede variar la incidencia específica en base a la edad de la paciente y de acuerdo a su genotipo, esto nos haría reevaluar la sugerencia que se desprende de estos estudios (32).

Si bien estos trabajos son concluyentes, nos parece importante destacar algunos puntos que nosotros consideramos relevantes al momento de evaluarlos. Si bien el estudio de Kauff y cols, demostró que la ooforectomía disminuye en conjunto el riesgo de cáncer de mama y ovario en $75 \%$, los resultados corresponden a un período de seguimiento muy corto (2 años), por lo que no es posible concluir con certeza el impacto real que tendrá el procedimiento sobre la incidencia del cáncer y en el riesgo acumulado de desarrollarlo a lo largo de la vida. Como método estimativo de lo anterior se seleccionó el cálculo del tiempo libre de cáncer a 5 años; sin embargo, el método tiene limitaciones pues no considera que la ooforectomía pudiera tener efectos adversos sobre el perfil lipídico y aumentar el riesgo de osteoporosis y enfermedades cardiovasculares, etc., repercutiendo sobre la calidad y expectativa de vida. Esto no pasaría si se considerara la sobrevida total después del procedimiento, pero estamos conscientes de que faltan muchos años para obtener esos datos.

En el estudio de Kauff y cols, podría haber un potencial sesgo como resultado de que excluyeron del análisis los 3 casos de cáncer de ovario en etapa temprana que fueron diagnosticados en el momento de la cirugía, pero incluyeron en éste potenciales casos de cánceres en etapas precoces en el grupo de vigilancia. El artículo muestra que los 5 cánceres ováricos o peritoneales desarrollados en el grupo de vigilancia, se diagnosticaron durante los primeros 8 meses de seguimiento y que en el grupo de las ooforectomizadas no hubo casos de cáncer en ese período. Esa obser- vación es consistente con un sesgo, debido a que se puede afirmar casi con certeza que estos 5 tumores estaban ya presentes al comienzo del período de seguimiento. De este modo se habría postergado la detección de los tumores en el grupo de vigilancia. La exclusión de los pacientes con cánceres preexistentes en un solo grupo de estudio, en un trabajo diseñado para determinar la incidencia de cáncer, sesga los resultados sustancialmente, en especial cuando el período de seguimiento es tan corto. Según Whitfield y cols (33) el método correcto hubiese sido incluir en el análisis a las 3 mujeres que se les diagnosticó el cáncer en el momento de la cirugía. Sin embargo, si esos 3 casos hubieran sido incluidos, el cociente de riesgo para desarrollar cáncer de mama u ovario seguiría siendo estadísticamente significativo $(0,37 ; 95 \%$ intervalo de confianza, 0,12 a 0,90$)$; otras conclusiones como el porcentaje de mujeres libres de cáncer a 5 años, hubiesen sido más débiles y menos categóricas.

Los estudios de modelos teóricos de análisisdecisión (22-25), concluyen, sobre la base de un rango estimado de la incidencia de cáncer, el pronóstico y la eficacia de la ooforectomía bilateral profiláctica. Este procedimiento otorgaría una ganancia en la expectativa de vida de entre 0,3 a 9,5 años. Ahora, como todo modelo matemático, es vital revisar críticamente todas las suposiciones que realizaron los autores para elaborarlos, especialmente a cómo ellos muestran la ganancia en expectativa de vida de forma muy variada de acuerdo a los supuestos, sobre todo aquellos relacionados con el riesgo de cáncer en pacientes con mutaciones en genes BRCA, la historia natural del cáncer de mama y ovario, la eficacia de la cirugía y las cifras de recurrencia. Así la base de algunos de esos supuestos no serían sólidas, ya que los modelos asumen que todos los tumores son biológicamente iguales y similares a aquellos que aparecen en pacientes portadoras de las mutaciones en los genes BRCA; no considera otros riesgos de salud que pudiesen afectar la expectativa de vida y asume además que las mujeres que poseen una mutación en los genes BRCA están informadas y saben su condición de portadoras (34).

Peshkin y cols (35), en una carta publicada en el New England Journal of Medicine, reconocen que, si bien ambos trabajos de Kauff y Rebbeck demuestran que los riesgos de cáncer de mama y ovario disminuyen significativamente después de la ooforectomía profiláctica y que los datos reportados por el último muestran un riesgo residual de 
cáncer de ovario muy bajo (5\%), el riesgo residual de cáncer de mama permanece aún muy alto (47\%). Basado en esto último, justifica medidas de manejo adicionales para el cáncer de mama (vigilancia, quimioprofilaxis y hasta mastectomía profiláctica en casos extremos). Destaca también que el mayor beneficio de la ooforectomía en términos de disminución del riesgo de cáncer de mama, fue descrito en mujeres premenopáusicas con mutaciones de los genes BRCA y sin historia previa de cáncer de mama, enfatizando que Rebbeck y cols no reportan los efectos de la ooforectomía profiláctica entre pre y postmenopáusicas y que las mujeres con edad mayor o igual a 50 años el riesgo de cáncer fue 0,52 (95\% intervalo de confianza 0,10 a 2,7). Así, según su opinión, a las mujeres postmenopáusicas no se les debería informar de que la ooforectomía profiláctica es efectiva en la disminución del riesgo de cáncer de mama, y se debería informar que la ooforectomía reduce principalmente el riesgo de cáncer de ovario y que podría reducir el riesgo de cáncer de mama en algunas mujeres.

Es importante tener presente las complicaciones asociadas a la cirugía, que son de baja frecuencia. La principal consecuencia negativa de la ooforectomía en mujeres premenopáusicas es la insuficiencia ovárica iatrogénica, que puede asociarse a osteoporosis, enfermedades cardiovasculares, bochornos, sequedad vaginal, disfunciones sexuales, alteraciones del sueño y cambios cognitivos que, en conjunto, producen una notable disminución en la calidad de vida (36). Un estudio reciente sugiere que las mujeres con este tipo de cirugía tienen más síntomas físicos y emocionales que las sometidas a vigilancia (37), este riesgo estaría compensado si se considera la morbimortalidad del cáncer y el posible tratamiento de los síntomas con medicamentos hormonales y no hormonales. Aunque las opiniones son divididas y la evidencia es poco clara sobre el uso de hormonoterapia de reemplazo (HTR) una vez realizada la ooforectomía profiláctica, la decisión debería tomarse teniendo en cuenta los síntomas que afecten la salud y su repercusión en la calidad de vida. Así, el capítulo sobre la administración de HTR en mujeres portadoras de mutaciones en los genes BRCA ooforectomizadas permanece abierto. Lo interesante es que en el estudio de Rebbeck y cols, las pacientes se beneficiaron con la ooforectomía pese a estar con HTR, coincidiendo con reportes previos y sugiriendo que el efecto protector de la cirugía no sería abolido por los preparados hormonales (21).

\section{CONCLUSIONES}

Los artículos revisados muestran evidencia a favor de la salpingo-ooforectomía profiláctica bilateral en portadoras de mutaciones BRCA, como una medida efectiva, tanto en la reducción del riesgo para cáncer de ovario y mama, como en el aumento de la expectativa de vida. Pensamos que este procedimiento debería reservarse para pacientes con fertilidad cumplida, entre 35 a 40 años de edad, con pesquisa periódica y precoz de cáncer mamario.

La efectividad de la ooforectomía profiláctica en portadoras de mutaciones BRCA otorga una poderosa razón a favor de la pesquisa genética en mujeres con historia familiar de cáncer de mama u ovario $\mathrm{y}$, aunque claramente falta tiempo para poder evaluar el impacto real a largo plazo de la ooforectomía profiláctica en la sobrevida total de las pacientes portadoras de genes BRCA mutados, creemos que existe evidencia suficiente como para justificar la ooforectomía profiláctica dentro de los protocolos de oncología preventiva en estas mujeres. Debemos procurar siempre que pese al rápido advenimiento de la genética al campo de la oncología, con su enorme potencial de prevención, diagnóstico y tratamiento, éste no sea sobrevalorado y transforme esas acciones en una suerte de cálculos de riesgo y probabilidades en desmedro de la atención y cuidado individual de nuestras pacientes.

\section{BIBLIOGRAFÍA}

1. Struewing JP, Hartge $P$, Wacholder S, Baker SM, Berlin M, McAdams $M$ y cols. The risk of cancer associated with specific mutations of BRCA1 and BRCA2 among Ashkenazi Jews. N Engl J Med 1997; 336: 20: 1401-8.

2. Satagopan JM, Boyd J, Kauff ND, Robson M, Scheuer L, Narod S y cols. Ovarian cancer risk in Ashkenazi Jewish carriers of BRCA1 and BRCA2 mutations. Clin Cancer Res 2002; 8(12): 3776-81.

3. Wooster R, Weber BL. Breast and ovarian cancer. N Engl J Med 2003; 348(23): 2339-47.

4. Thull DL, Vogel VG. Recognition and Management of Hereditary Breast Cancer Syndromes. Oncologist 2004; 9(1): 13-24.

5. De Fazio A. New evidence on the function of BRCA1 and BRCA2. Newsletter of the NHMRC. National Breast Cancer Centre 1998; 3(4): 3-5.

6. Easton DF, Ford D, Bishop DT. Breast and ovarian cancer incidence in BRCA1- mutation carriers. Am J Hum Genet 1995; 56(1): 265-71. Abstract.

7. Hopper JL. Some public health issues in the current 
state of genetic testing for breast cancer in Australia. Australian and New Zealand Journal of Public Health. 1996; 20(5): 467-72.

8. Meijers-Heijboer EJ, Verhoog LC, Brekelmans CT, Seynaeve C, Tilanus-Linthorst MM, Wagner A, y cols. Presymptomatic DNA testing and prophylactic surgery in families with BRCA1 or BRCA2 mutations. Lancet 2000; 355(9220): 2015-20.

9. Scheuer L, Kauff N, Robson M, Kelly B, Barakat R, Satagopan $\mathrm{J}$ y cols. Outcome of preventive surgery and screening for breast and ovarian cancer in BRCA mutations carriers. J Clin Oncol 2002; 20(5): 1260-8.

10. Schwartz MD, Kaufman E, Peshkin BN, Isaacs C, Hughes C, DeMarco T y cols. Bilateral prophylactic oophorectomy and ovarian cancer scrrening following BRCA1/BRCA2 mutation testing. J Clin Oncol 2003; 21(21): 4034-41.

11. Helzlsouer K. Bad news/Good News: Information about breast cancer risk following prophylactic oophorectomy. J Natl Cancer Inst 1999; 91(17): 1442-3.

12. Levy-Lahad E, Catane R, Eisenberg S, Kaufman B, Hornreich G, Lishinsky E y cols. Founder BRCA1 and BRCA2 mutations in Ashkenazi Jews in Israel: frequency and differential penetrance in ovarian cancer and in breast-ovarian cancer. Am J Hum Genet 1997; 60(5): 1059-67. Abstract.

13. Haber D. Prophylactic oophorectomy to reduce the risk of ovarian and breast cancer in carriers of BRCA mutations. N Engl J Med 2002; 346 (21):1660-2.

14. Pichert G, Bolliger B, Buser K, Pagani O. Evidencebased management options for women at increased breast/ovarian cancer risk. Ann Oncol 2003; 14(1): 9-19.

15. Lu KH, Garber JE, Cramer DW, Welch WR, Niloff J, Schrag $D$ y cols. Occult ovarian tumors in women with BRCA1 or BRCA2 mutations undergoing prophylactic oophorectomy. J Clin Oncol 2000; 18(14): 2728-32.

16. Eisen A, Rebbeck TR, Wood WC, Weber BL. Prophylactic surgery in women with a hereditary predisposition to breast and ovarian cancer. J Clin Oncol 2000; 18(9): 1980-95. Abstract.

17. Struewing JP, Watson P, Easton DF, Ponder BA, Lynch HT, Tucker MA. Prophylactic oophorectomy in inherited breast/ovarian cancer. J Natl Cancer Inst Monogr 1995; 17: 33-5. Abstract.

18. Rebbeck TR, Levin AM, Eisen A, Snyder C, Watson $P$, Cannon-Albright $L$ y cols. Breast cancer risk after bilateral prophylactic oophorectomy in BRCA1 mutations carriers. J Natl Cancer Inst 1999; 91(17): 14759.

19. Rebbeck TR, Prophylactic oophorectomy in BRCA1 and BRCA2 mutation carriers. J Clin Oncol 2000; 18(21 Supl): 100S-3S.

20. Kauff ND, Satagopan JM, Robson ME, Scheuer L,
Hensley M, Hudis CA y cols. Risk-reducing salpingo -oophorectomy in women with a BRCA1 or BRCA2 mutation. N Engl J Med 2002; 346(21): 1609-15.

21. Rebbeck TR, Lynch HT, Neuhausen SL, Narod SA, Van't Veer L, Garber JE y cols. Prevention and Observation of Surgical End Points Study Group. Prophylactic oophorectomy in carriers of BRCA1 or BRCA2 mutations. New Engl J Med 2002; 346(21): 1616-22.

22. Schrag D, Kuntz KM, Garber JE, Weeks JC. Decision analysis-effects of prophylactic mastectomy and oophorectomy on life expectancy among womwn with BRCA1 and BRCA2 mutations. New Engl J Med 1997; 336(20): 1465-71.

23. van Roosmalen MS, Verhoef LC, Stalmeier PF, Hoogerbrugge N, van Daal WA. Decision analysis of prophylactic surgery or screening for BRCA1 mutations carrier: A more prominent role for oophorectomy. J Clin Oncol 2002; 20(8): 2092-100.

24. Grann VR, Panageas KS, Whang W, Antman KH, Neugut Al. Decision analysis of prophylactic mastectomy and oophorectomy in BRCA1-positive and BRCA2-positive patines. J Clin Oncol 1998; 16(3): 979-85.

25. Grann VR, Jacobson JS, Thomason D, Hershman D, Heitjan DF, Neugut Al. Effect of prevention strategies on survival and quality-adjusted survival of women with BRCA 1/2 mutations: An updated decision analysis. J Clin Oncol 2002; 20(10): 2520 9.

26. Fong YF, Lim FK, Arulkumaram S: Prophylactic Oophorectomy. A continuing controversy. Obstet Gynecol Surv 1998; 53(8): 493-99.

27. Aziz S, Kuperstein G, Rosen B, Cole D, Nedelcu R, McLaughlin $\mathrm{J}$ y cols. A genetic epidemiological study of carcinoma of the fallopian tube. Gynecol Oncol 2001; 80(3): 341-5.

28. Piek JM, van Diest PJ, Zweemer RP, Jansen JW, Poort-Keesom RJ, Menko FH y cols. Dysplastic changes in prophylactically removed fallopian tubes of women predisposed to developing ovarian cancer. J Pathol 2001; 195(4): 451-6.

29. Paley PJ, Swisher EM, Garcia RL, Agoff SN, Greer $\mathrm{BE}$, Peters KL y cols. Occult cancer of the fallopian tube in BRCA-1 germline mutation carriers at prophylactic oophorectomy: a case for recommending hysterectomy at surgical prophylaxis. Gynecol Oncol 2001; 80(2): 176-8.

30. Rubin SC, Benjamin I, Behbakht K, Takahashi H, Morgan MA, LiVolsi VA y cols. Clinical and pathological features of ovarian cancer in women with germline mutation of BRCA1. N Engl J Med 1996; 335(19): 1413-6.

31. NIH Consensus Development Panel on Ovarian Cancer. NIH consensus conference. Ovarian cancer. Screening, treatment, and follow up. JAMA 1995; 273(6): 490-97. 
32. Anderson WF. Oophorectomy in carriers of BRCA mutations. N Engl J Med 2002; 347(13): 1037-40. (Carta al editor)

33. Whitfield GA. Oophorectomy in carriers of BRCA mutations. N Engl J Med 2002; 347(13): 1037-40. (Carta al editor)

34. McCaffrey J. Effects of prophylactic surgery. Newsletter of the NHMRC National Breast Cancer Centre 1998; 2(4): 4-6.
35. Peshkin BN. Oophorectomy in carriers of BRCA mutations. New Engl J Med 2002; 347(13): 1037-40. (Carta al editor)

36. Ahmad K. Oophorectomy reduces cancer risk. Lancet Oncol 2002; 3(7): 393-4.

37. Fry A, Busby-Earle C, Rush R, Cull A. Prophylactic oophorectomy versus screening: psychosocial outcomes in women at increased risk of ovarian cancer. Psychooncology 2001; 10(3): 231-41. Abstract. 